\title{
Research on a Conflict Early Warning System Based on the Active Safety Concept
}

\author{
Bozhao Shen, ${ }^{1}$ Zhiqing Zhang $\mathbb{D D}^{2}{ }^{2}$ Hao Liu, ${ }^{1}$ Shiyun Li, ${ }^{1}$ and Lei Zhao ${ }^{1}$ \\ ${ }^{1}$ College of Metropolitan Transportation, Beijing University of Technology, No. 100 Pin Le Yuan, Chao Yang District, \\ Beijing 100124, China \\ ${ }^{2}$ Engineering Research Center of Urban Transportation, Beijing University of Technology, No. 100 Pin Le Yuan, \\ Chao Yang District, Beijing 100124, China
}

Correspondence should be addressed to Zhiqing Zhang; zhangzhiqing@bjut.edu.cn

Received 27 January 2018; Revised 6 June 2018; Accepted 3 July 2018; Published 12 August 2018

Academic Editor: Aboelmaged Noureldin

Copyright (C) 2018 Bozhao Shen et al. This is an open access article distributed under the Creative Commons Attribution License, which permits unrestricted use, distribution, and reproduction in any medium, provided the original work is properly cited.

In order to reduce traffic conflicts on cross-intensive roads, this paper proposes a new early warning system based on the active safety concept. The system collects real-time vehicle data using roadside sensors and transmits the results to drivers on the major road in a timely manner via roadside warning lights. In this research, the principles of the warning system are discussed in detail, including how the vehicle dynamics data are collected and how potential collisions are identified and avoided. Through a driving simulation experiment, the speed prediction model after implementation of the warning system was examined. Results indicated that it can accurately identify the vehicle operating status, accurately guide driving behavior, and effectively reduce traffic conflict. To verify the reliability of the proposed warning logic and algorithm, numerical simulations were carried out via CarSim/Simulink cosimulation. The simulation results indicate that the proposed system enables drivers to perceive conflicting vehicles in advance, avoid the sudden braking phenomenon, and ensure safe driving.

\section{Introduction}

In China, many national and provincial roads have numerous minor roads intersecting them and carry mixed traffic. With a lack of management and oversight, the existing safety facilities make it difficult for drivers to behave safely. According to statistics, in 2015, the accident rate at intersections on China's national and provincial roads was $34.21 \%$, and the majority of accidents were caused by a lack of sight distance [1].

In order to reduce traffic accidents and casualties, a variety of traffic safety facilities have been installed at highway intersections. The most common of these facilities is a continuously flashing warning light. However, the presence of many road intersections and little traffic flow on minor roads renders the continuous flashing warning lights ineffective. In most cases, the minor roads carry no vehicles; therefore, the frequent warnings are often ignored by drivers on the major roads. Thus, the existing warning light system does not address the safety issues associated with multiple-intersection roads; a new warning system is urgently needed.
With the development of the Internet of Vehicles (IOV), many scholars have begun to consider improving the safety and security of intersections through vehicle-to-vehicle communication technologies [2, 3]. Joyoung Lee et al. [4] proposed a vehicle cooperative control algorithm that could effectively reduce the stopping frequency and travel time through unsignalized intersections. Ismail H. et al. [5] proposed a vehicle coordination adaptive cruise control (ACC) system based on the game theory optimization algorithm. Fuerstenberg proposed an intersection-collision warning system which can trigger a conflict warning when a vehicle collision is detected [6]. Liu Jiang [7] proposed a collaborative vehicle collision warning system, based on the relative speed and direction of the vehicles. Shih-Yang Lin [8] proposed a vehicle cooperative collision warning algorithm that could adapt to various speeds. However, IOV technology is neither universal nor mature, and pedestrians and nonmotor vehicles are not factored into the network environment. Vehicle-tovehicle communication alone does not ensure the safety of all traffic on cross-intensive roads. Therefore, a new approach 
is urgently needed to reduce intersection conflicts for all users.

Driver behavior is a significant factor in traffic accidents and has prompted extensive and in-depth study by scholars $[9,10]$. To verify the effectiveness of warning systems, their effect on driver behavior under different driving environments can be analyzed. By collecting data related to driver collision avoidance behavior, Sun D. et al. [11] estimated collision avoidance probability and described emergency avoidance behavior. Through the analysis of avoidance behavior, Gorjestani et al. [12] determined that setting warning signs at a certain position can reduce collision accidents by up to $35 \%$. These studies analyzed driving behavior in collision avoidance scenarios but did not account for the effect of complex traffic environments on collision avoidance behavior. Driving simulation experiments are a feasible way to eliminate confound from different traffic environments across similar studies $[13,14]$. Using a driving simulator, Horst et al. [15] analyzed the effect of road facilities on driving speed and vehicle transverse position.

The development of image processing and electronic communication technology has made it possible to obtain vehicle information accurately and simultaneously. Kihei et al. [16] proposed an architecture that uses omnidirectional antennas to achieve 3D localization and employs Doppler domain analysis to determine the threat of collision. Toledo R. [17] presented a maneuver prediction that employs a Global Navigation Satellite System (GNSS) receiver, one gyro, one accelerometer, and the odometry. Liu Jiang et al. [18] proposed a novel cooperative vehicle conflict resolution algorithm to predict unsignalized intersection collisions based on an enhanced road map. In order to determine the location, speed, and other vehicle information, the above studies used a variety of advanced equipment, such as enhanced road maps, omnidirectional antennas, and accelerometers. However, considering the cost, real-time signal requirements, and system reliability, the above methods cannot be widely applied to cross-intensive roads.

In summary, the existing technologies and facilities are inadequate to mitigate safety concerns and collision incidents on cross-intensive roads in China. Therefore, this paper proposes a new conflict warning system to enable drivers perceive intersection conflicts sooner and effectively avoid the conflicts. There are three major objectives in this article: (1) introduce the working principles of the warning system and the calculation method of the conflict resolution model; (2) evaluate the effectiveness of the proposed system through driving simulation experiments; and (3) verify the reliability of the proposed warning logic and algorithm through CarSim/Simulink cosimulation.

\section{Methods}

2.1. Overall Design. Based on the active safety concept, the intersection warning system combines warning lights with roadside detectors. This system can collect real-time vehicle data and transmits the results to drivers in a timely manner via the warning lights.
2.1.1. Basic Composition. The warning system consists of a sensor warning light and two vehicle detectors.

(i) Sensor warning light: the sensor warning light is installed prior to the major road intersection and has two states: continuously flashing or off. When no conflict is detected, the warning light remains off, meaning that vehicles may pass safely. When the system determines that a conflict exists, the warning light flashes continuously, informing drivers that the next intersection may have a conflict risk and to proceed with caution.

(ii) Vehicle detectors: the two detectors are installed adjacent to the intersection in order to detect vehicles approaching from both directions on the main and minor roads. The detectors record the vehicle speeds and positions and transfer the data to the system in real time.

2.1.2. Basic Framework. The framework of the warning system consists of four parts: data collection, situation perception, risk assessment, and decision execution (see Figure 1). The specific detection process is as follows.

(1) Data Collection. The radar detectors installed adjacent to the intersection collect real-time data on both location and speed of the nearest vehicle.

(2) Situation Perception. When vehicles are simultaneously detected on both roads, the system will calculate the time until the conflict point, known as the Time to Collision (TTC).

(3) Risk Assessment. Based on the TTC, the system will evaluate whether or not there is the potential for a collision. For safety purposes, drivers' reaction times are incorporated into the algorithm.

(4) Decision Execution. Once a potential conflict is predicted, a warning signal will be issued to the road that does not have right of way. The light will not be deactivated until the algorithm confirms that a safe path is available.

2.2. Theoretical Analysis. Conflict identification is key to the efficacy of the warning system. The conflict identification theory and algorithm is described in detail in this section.

2.2.1. Data Collection. Precise vehicle location is essential. Radar detectors are a low cost and efficient speed detecting system [19]. They can obtain effective data with sufficient precision.

To locate vehicles relative to the road intersection, a Cartesian coordinate system is established with the center of collision zone as the original point (Figure 2). Based on the placement position and angle of the detector, the calculation formulas for the radar detection range are

$$
X_{1}=X_{0}-\frac{H}{\tan (\theta+\beta / 2)}
$$


DATA

COLLECTION

RISK

SITUATION

RECEPTION

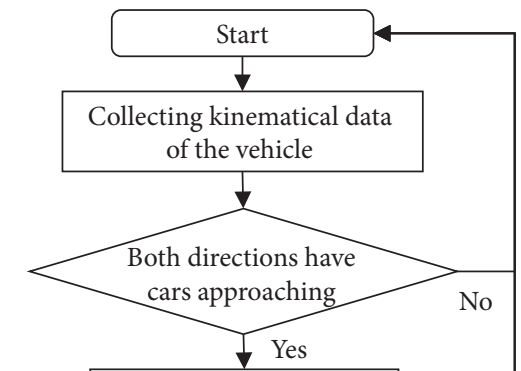

Predict the time to the collision zone

ASSESSMENT

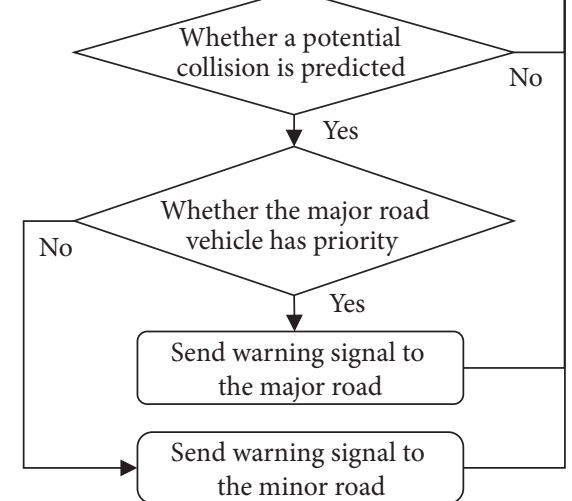

FIGURE 1: Hierarchical framework of the warning system.

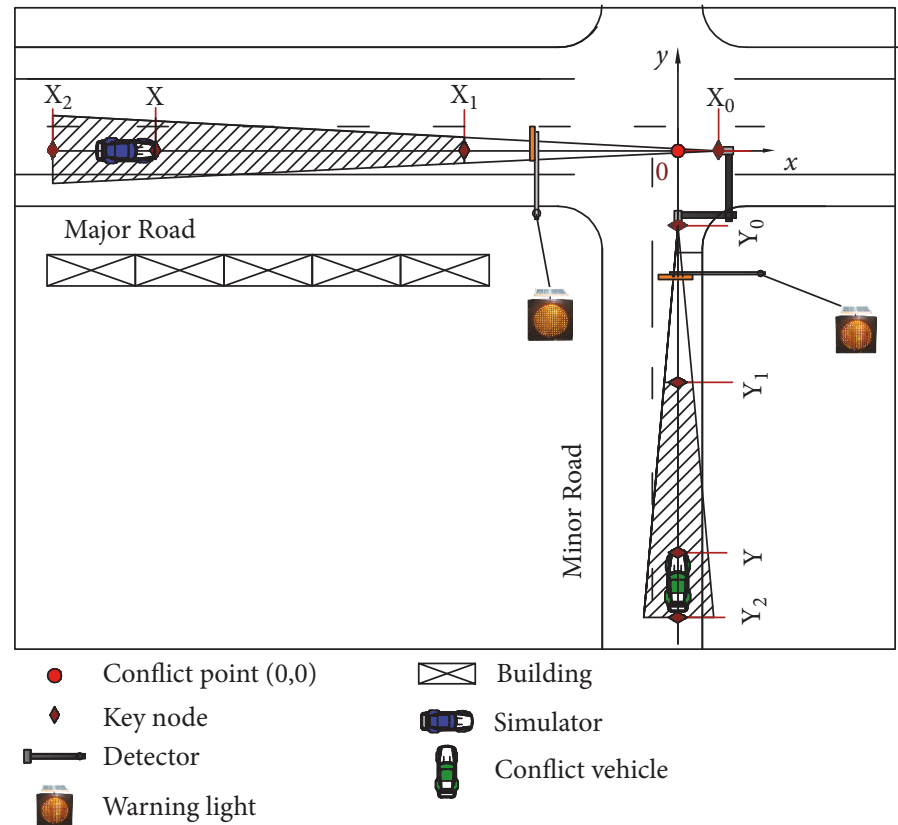

FIGURE 2: Design diagrams of the warning system.

$$
\begin{gathered}
X_{2}=X_{0}-\frac{H}{\tan (\theta-\beta / 2)} \\
D=\frac{\tan \alpha}{\sin (\theta+\beta / 2)} H,
\end{gathered}
$$

where $H$ is height of the detector, $\theta$ is vertical mounting angle of the radar detector, $\beta$ is radiation angle of the radar beam; $\beta \leq 7^{\circ}$ generally meets the accuracy requirements [20], D is width of horizontal detection zone, $\mathrm{X}_{0}\left(\mathrm{Y}_{0}\right)$ is relative position from conflict point to major road (minor road) detector, $\mathrm{X}_{1}$ $\left(\mathrm{Y}_{1}\right)$ is minimum distance from conflict point to the detection 


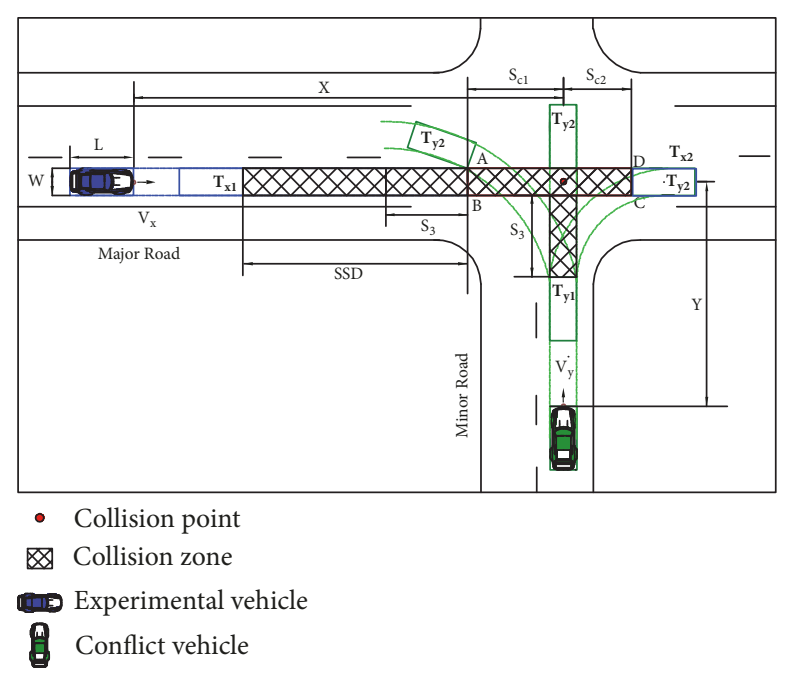

FIgURE 3: Intersection-collision zone.

zone, and $\mathrm{X}_{2}\left(\mathrm{Y}_{2}\right)$ is maximum distance from conflict point to the detection zone.

According to the actual road conditions and the required detection range, the values of the above parameters are defined as $\beta=7^{\circ}, \mathrm{H}=5.5 \mathrm{~m}, \mathrm{D}_{\text {min }}=3.75 \mathrm{~m}, \mathrm{X}_{0}=5 \mathrm{~m}$, and $\mathrm{Y}_{0}=-8 \mathrm{~m}$. Thus, the major road detection zone is within the range from $X_{2}=-150 m$ to $X_{1}=-30 m$, and the minor road detection zone is from $Y_{1}=-120 m$ to $Y_{1}=-40 m$.

\subsubsection{Situation Perception}

(1) Stopping Sight Distance. Stopping sight distance (SSD) [21] is the minimum sight distance required for the driver to stop a vehicle traveling safely without collision with any obstruction. As the angle of the conflict vehicles is $90^{\circ}$, for major road vehicles, the velocity component in the $\mathrm{Y}$ direction is 0 . Therefore, the stopping sight distance between two vehicles can be solved based on the model relative to stationary objects. The specific formula is

$$
S S D=\frac{V}{3.6} t+\frac{V^{2}}{254(f \pm g)}+S_{3},
$$

whereSSD is stopping sight distance required for vehicles, $\mathrm{m}, \mathrm{t}$ is perception-reaction time, sec., typically $2.5 \mathrm{~s}$ [22], $\mathrm{f}$ is coefficient of friction; for a poor, wet pavement, this is typically $0.31-0.44, \mathrm{~S}_{3}$ is safe interval of the stationary vehicle; generally 5-10 $\mathrm{m}$, and $\mathrm{g}$ is grade, decimal.

In order to ensure safety, we set $S_{3}=10 \mathrm{~m}$. Within the allowable range of error, (2) can be simplified to

$$
S S D=1.25 \mathrm{~V} .
$$

(2) Time to Collision (TTC). TTC is the time required for the major road (minor road) vehicle to reach the collision point. This parameter was introduced to predict whether or not approaching vehicles will come into conflict.

Based on three different vehicle trajectories, the dangerous intersection zone is defined as 'ABCD', as shown in
Figure 3. To ensure safety, the distance from the major road car to the conflict area must meet the SSD when the minor road car arrives at the intersection. Therefore, the dangerous intersection zone of the two directions was expanded, as shown in the red area in Figure 3. When it is predicted that the two cars will be in the collision zone at the same time, there is a risk of conflict.

The time required for the major road vehicles to enter and leave the collision zone can be calculated through the following formulas:

$$
\begin{aligned}
& T_{x 1}=T T C_{x}-\frac{3.6}{V_{x}}\left(S_{c 1}+S S D\right) \\
& T_{x 2}=T T C_{x}+\frac{3.6}{V_{x}}\left(S_{c 2}+L\right) \\
& T_{y 1}=T T C_{y}-\frac{3.6}{V_{y}}\left(\frac{W}{2}+S_{3}\right) \\
& T_{y 2}=T T C_{y}+\frac{3.6}{V_{y}}\left(\frac{W}{2}+L\right)+\delta,
\end{aligned}
$$

where $\mathrm{T}_{\mathrm{x} 1}\left(\mathrm{~T}_{\mathrm{y} 1}\right)$ is time required for the major (minor) road vehicle to enter the collision zone, $\mathrm{T}_{\mathrm{x} 2}\left(\mathrm{~T}_{\mathrm{y} 2}\right)$ is time required for the major (minor) road vehicle to leave the collision zone, $\mathrm{L}$ is length of the vehicle; typically $3.8-4.3 \mathrm{~m}$, and assumed to be $\mathrm{L}=4 \mathrm{~m}$ in this case, $\mathrm{W}$ is width of the vehicle, typically $1.6-1.8 \mathrm{~m}$, and assumed to be $\mathrm{W}=2 \mathrm{~m}$ in this case, $\mathrm{S}_{\mathrm{cl}}$ is distance from the left-turn collision zone boundary to the conflict point, $\mathrm{S}_{\mathrm{c} 2}$ is distance from the right turn collision zone boundary to the conflict point, and $\delta$ is the left-right deviation coefficient, assumed to be $0 \sim 3 \mathrm{~s}$.

2.2.3. Risk Assessment. According to the above, when one of the following two situations is satisfied, the warning logic will be activated. 
TABLE 1: Minimum acceptable gap for vehicles through an intersection.

\begin{tabular}{|c|c|c|c|c|}
\hline \multirow{2}{*}{ Direction } & \multicolumn{4}{|c|}{ Minimum acceptable gap (Tc) } \\
\hline & Small car & Medium car & Large car & Trailer \\
\hline Turn left & $5.77 \mathrm{~s}$ & $6.91 \mathrm{~s}$ & $7.48 \mathrm{~s}$ & $8.05 \mathrm{~s}$ \\
\hline Go straight & $4.81 \mathrm{~s}$ & $5.77 \mathrm{~s}$ & 7.11s & $7.18 \mathrm{~s}$ \\
\hline Turn right & $2.94 \mathrm{~s}$ & $3.69 \mathrm{~s}$ & $4.15 \mathrm{~s}$ & $4.62 \mathrm{~s}$ \\
\hline
\end{tabular}

(1) $T_{x 1} \leq T_{y 1} \leq T_{x 2}$. The major road vehicle arrives at the collision zone first, and when the minor road vehicle arrives, the major road vehicle has not completely left the collision zone. The specific formula is

$$
\begin{aligned}
& T^{T} C_{x}-\frac{3.6}{V_{x}}\left(S_{c 1}+S S D\right) \leq T T C_{y}-\frac{3.6}{V_{y}}\left(\frac{W}{2}+S_{3}\right) \\
& \quad \leq T T C_{x}+\frac{3.6}{V_{x}}\left(S_{c 2}+L\right) .
\end{aligned}
$$

(2) $T_{y 1} \leq T_{x 1} \leq T_{y 2}$. The minor road vehicle arrives at the collision zone first, and when the major road vehicle arrives, the minor road vehicle has not completely left the collision zone. The specific formula is

$$
\begin{aligned}
& T^{T} C_{y}-\frac{3.6}{V_{y}}\left(\frac{W}{2}+S_{3}\right) \leq T T C_{x}-\frac{3.6}{V_{x}}\left(S_{c 1}+S S D\right) \\
& \leq T T C_{y}+\frac{3.6}{V_{y}}\left(\frac{W}{2}+L\right)+\delta .
\end{aligned}
$$

By simplifying and integrating (5) and (6), the conflict threshold is obtained:

$$
-\frac{14}{V_{x}} \leq \frac{|X|-6}{V_{x}}-\frac{|Y|-6}{V_{y}} \leq \frac{11}{V_{y}}+2 .
$$

When the detected data satisfies the conflict threshold (see (7)), the intersection has a potential conflict risk.

2.2.4. Decision Execution. Through a large number of traffic observations at different regional intersections in China, the minimum acceptable gap required for vehicles crossing an intersection was obtained, as shown in Table 1.

The priority decision formula for major road vehicles is shown in

$$
T T C_{\mathrm{x} 1}-T T C_{\mathrm{y} 1}<T_{c},
$$

whereTTC $\left(\mathrm{TTC}_{\mathrm{y}}\right)$ is time required for the major (minor) road vehicle to reach the collision point and $\mathrm{T}_{c}$ is minimum acceptable gap. To ensure safety, $\mathrm{T}_{\mathrm{c}}$ was assumed to be $6 \mathrm{~s}$.

Conflict warning lights are set on the major road and the minor road, respectively. When a potential conflict risk is detected, the system determines which road vehicle has priority and sends warning signals to the vehicle without priority. The warning light will not be deactivated until the algorithm confirms that a safe departure is available.

When the warning light turns off, the system will continue the following process:
(1) Continuously detect vehicle speeds and position information.

(2) If vehicles are detected in both directions simultaneously, estimate the TTC of the two vehicles.

(3) Determine whether or not the conflict threshold is reached.

(4) Judge the vehicle priority, and turn the warning light off or on as appropriate.

\section{Analysis and Results}

3.1. Effectiveness Evaluation. To analyze the traffic patterns after installing the warning system and to evaluate the effectiveness of the system, a driving simulation experiment was carried out.

\subsubsection{Driving Simulation Experiment}

(i) Subjects. In this experiment, 30 subjects were recruited. The subjects ranged in age from 25 to 45 years, with an average age of 32 years and an average driving experience of 6 years.

(ii) Apparatus. Driving simulator: the driving simulator used in this experiment was a real vehicle simulator which can provide realistic 3D driving scenarios and collect both vehicle operation data and driving behavior data.

(iii) Scenario. This experiment involved two scenarios (Figure 4). Specific scenario descriptions are shown in Table 2.

(iv) Principle. Most cross-intensive roads are second or third grade highways. Accordingly, the speed limit in this experiment was set to $60 \mathrm{~km} / \mathrm{h}$. At each intersection, whether or not the minor road was occupied by a conflict vehicle was randomized. In order to create an emergency collision situation, the speed of the minor road car was adjusted according to the speed of the major road car. In the warning light scenario, the warning system was installed at each intersection. When a minor road car approached the intersection, the warning light started flashing continuously. When there is no vehicle detected, the warning light was turned off.

(v) Procedures. The experimental procedure was as follows. (1) Fill out a basic information questionnaire. (2) Read the predriving instructions. Subjects were told that the speed limit was $60 \mathrm{~km} / \mathrm{h}$ and was informed of the display theory of the warning light. (3) Perform a test drive for five minutes. 
TABLE 2: Experiment scenario descriptions.

\begin{tabular}{lcc}
\hline Scenario & Standard group & Warning light group \\
\hline Description & Set intersection safety facilities by standard. & Standard group + Warning system \\
\hline
\end{tabular}

TABLE 3: Scores of driving authenticity.

\begin{tabular}{lcccccc}
\hline General level & $\begin{array}{c}\text { Steering } \\
\text { Wheel }\end{array}$ & Accelerator & Brake & Clutch & $\begin{array}{c}\text { Scenarios } \\
\text { perception }\end{array}$ \\
\hline 7.9 & 7.2 & 7.9 & 7.7 & 8.8 & 7.9 & 7.8 \\
\hline
\end{tabular}

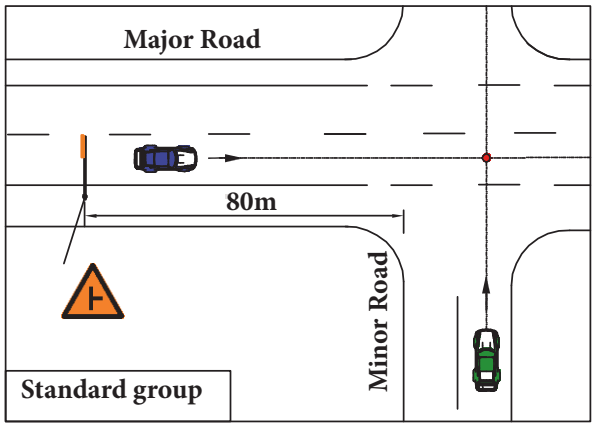

(a)

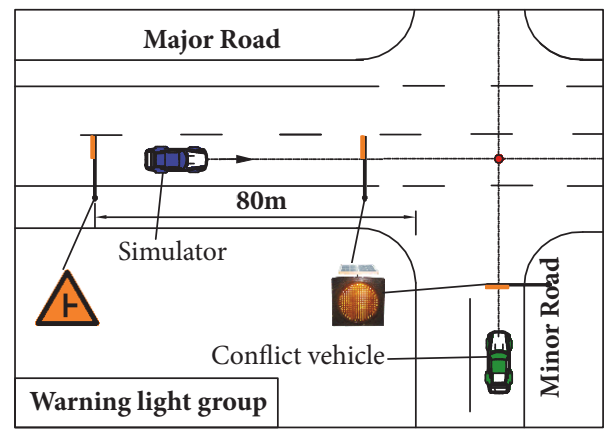

(b)

FIGURE 4: Description of the two scenarios: (a) standard group; (b) warning light group.

(4) Select six scenarios at random and drive the scenarios in turn. (5) Fill out a driving authenticity questionnaire.

3.1.2. Driving Authenticity Analysis. In order to verify the driving authenticity, subjects were asked to subjectively evaluate the performance of the driving simulator. The ratings ranged from 0 to 10 , where 0 represents completely different from the real road and 10 represents extreme similarity to the real road. The scores for the driving authenticity analysis are shown in Table 3.

These results show that most subjects found the driving simulation experiment to be similar to the real road environment in terms of both the driving scenarios and driving operation.

\subsubsection{Driving Behavior Analysis}

(i) Velocity Changes. Due to inconsistency in the minimum value of velocity, direct speed weighting may cause large errors. Therefore, the K-means clustering method was used to better reflect the trends in speed variation. K-means clustering is a simple and effective clustering algorithm [23]. After a classification and integration process, the velocity change curve is obtained (Figure 5(a)).

In order to quantitatively describe the continuity of the actual driving speed of the subjects, the concept of velocity deviation was introduced, which is represented by the symbol $\Delta v$.

$$
\Delta v=\sqrt{\frac{1}{n} \sum_{i=1}^{n}\left(v_{i+1}-v_{i}\right)^{2}}
$$

where $v_{i}$ is a speed of point $\mathrm{i}$ within the scope of the intersection data analysis, $v_{i+1}$ is a speed of point $\mathrm{i}+1$ within the scope of the intersection data analysis, and $n$ is the number of speed data points; in this experiment, speed data was obtained every $5 \mathrm{~m}$; thus, $\mathrm{n}=40$.

As shown in Figure 5(a), the mean value of the minimum speed for the warning light group was about $35.63 \mathrm{~km} / \mathrm{h}$, while in the standard group it was only $12.35 \mathrm{~km} / \mathrm{h}$ (Table 4 ). Meanwhile, the speed change in the warning light group was less than in the standard group. This means that, during the collision avoidance process, the warning system can effectively reduce the speed change rate and improve the speed continuity.

(ii) Acceleration Change. The K-means clustering method was also used to obtain the acceleration curve. As shown in Figure 5(b), the acceleration in the standard group changed significantly; the maximum deceleration was 6.43 $\mathrm{m} / \mathrm{s} 2$. However, in the warning light group, the acceleration remained relatively stable; the maximum deceleration was only $1.65 \mathrm{~m} / \mathrm{s} 2$ (Table 4 ). This means that when the warning system is implemented, subjects are able to initiate deceleration earlier and effectively avoid rapid deceleration.

(iii) Gas Pedal Change. Using the K-means clustering method, the gas pedal change curve was obtained. As shown in Figure 5(c), drivers in the warning light group released the gas pedal $35 \mathrm{~m}$ earlier than those in the standard group (Table 5), and the loose throttle rate in the warning light group was slower than in the standard group. This means that with the warning system, drivers are able to perceive the danger earlier and take deceleration measures more calmly during the conflict avoidance process. 
TABLE 4: Vehicle operation status under different warning scenarios.

\begin{tabular}{lccc}
\hline Index & Parameter & Standard group & Warning light group \\
\hline \multirow{2}{*}{ Velocity } & Minimum speed & $12.35 \mathrm{Km} / \mathrm{h}$ & $35.63 \mathrm{Km} / \mathrm{h}$ \\
& Velocity deviation & $3.35 \mathrm{~m} / \mathrm{s}$ & $1.03 \mathrm{~m} / \mathrm{s}$ \\
\hline \multirow{2}{*}{ Acceleration } & Maximum deceleration & $6.43 \mathrm{~m} / \mathrm{s} 2$ & $1.65 \mathrm{~m} / \mathrm{s} 2$ \\
& Acceleration standard deviation & $1.56 \mathrm{~m}$ & $0.51 \mathrm{~m}$ \\
\hline
\end{tabular}

TABLE 5: Driving behavior under different warning scenarios.

\begin{tabular}{lccc}
\hline Index & Parameter & Standard group & Warning light group \\
\hline \multirow{2}{*}{ Gas pedal } & Minimum accelerator position & $46.03 \mathrm{~m}$ & $11.4 \mathrm{~m}$ \\
& Rate of gas pedal aperture change & $57.66 \mathrm{~N} / \mathrm{s}$ & $81.09 \mathrm{~N} / \mathrm{s}$ \\
\hline \multirow{2}{*}{ Brake pedal } & Maximum braking force & $106.53 \mathrm{~N}$ & $47.21 \mathrm{~N}$ \\
& Maximum braking position & $37.21 \mathrm{~m}$ & $51.87 \mathrm{~m}$ \\
\hline
\end{tabular}

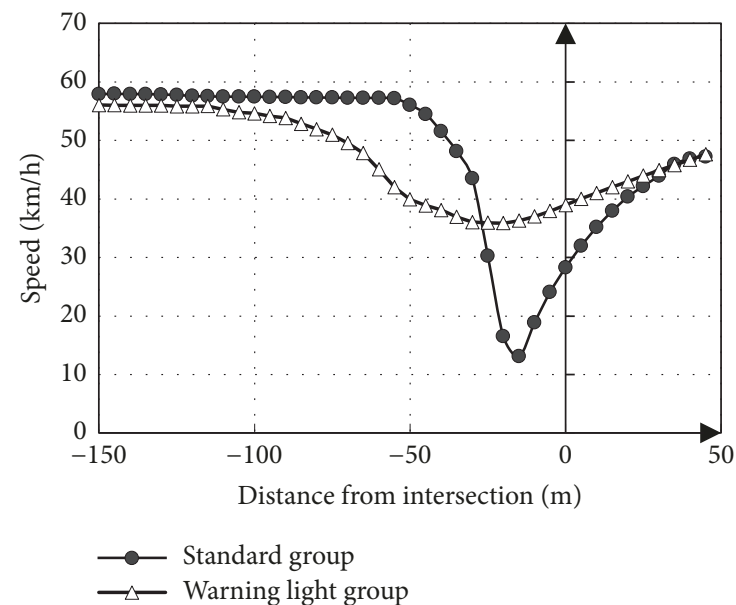

(a)

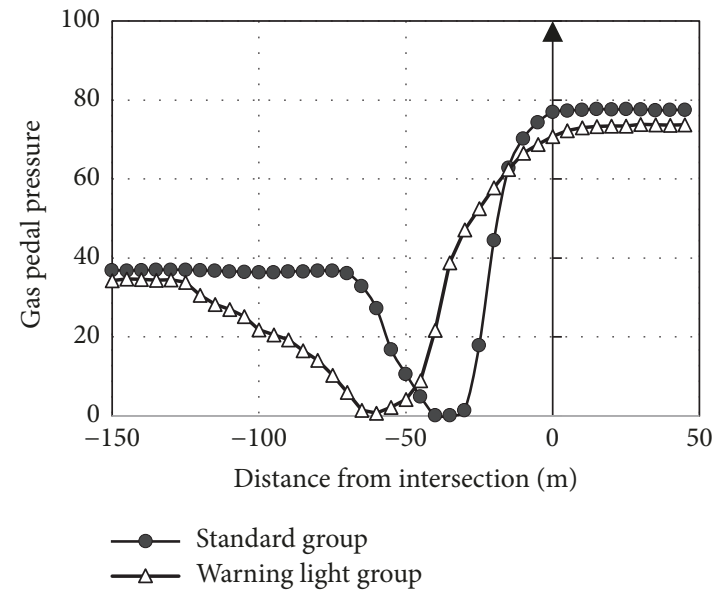

(c)

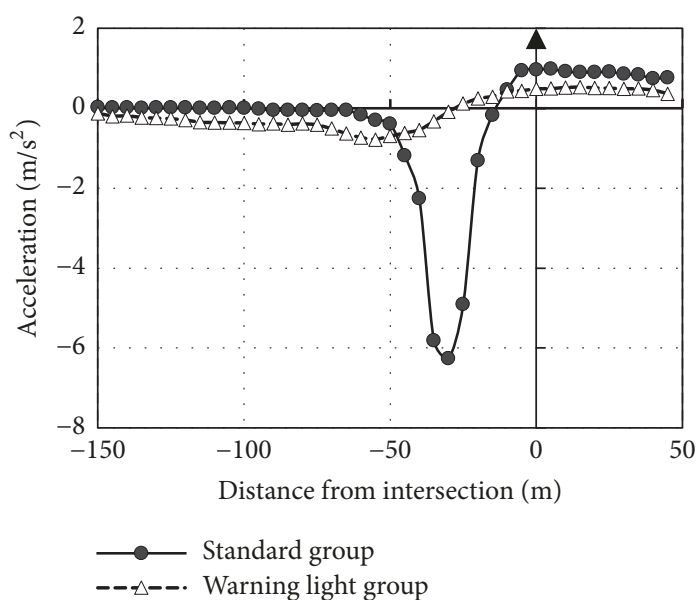

(b)

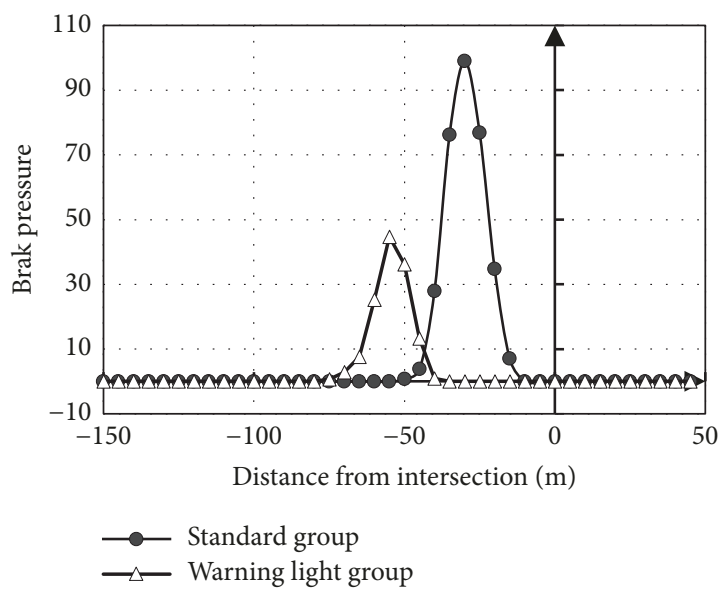

(d)

FIGURE 5: Experiment result: (a) velocity change; (b) acceleration change; (c) gas pedal change; (d) brake pedal change.

(iv) Brake Pedal Change. Using the K-means clustering method, brake pedal data was collated by taking the maximum brake pedal pressure. As shown in Figure 5(d), the maximum braking power of the warning light group was only half that of the standard group (Table 5). These results show that the warning system can effectively reduce the maximum braking power and avoid emergency braking behavior.

As can be seen from the experimental results, during the collision avoidance process, the warning system can effectively improve the speed continuity and avoid rapid 


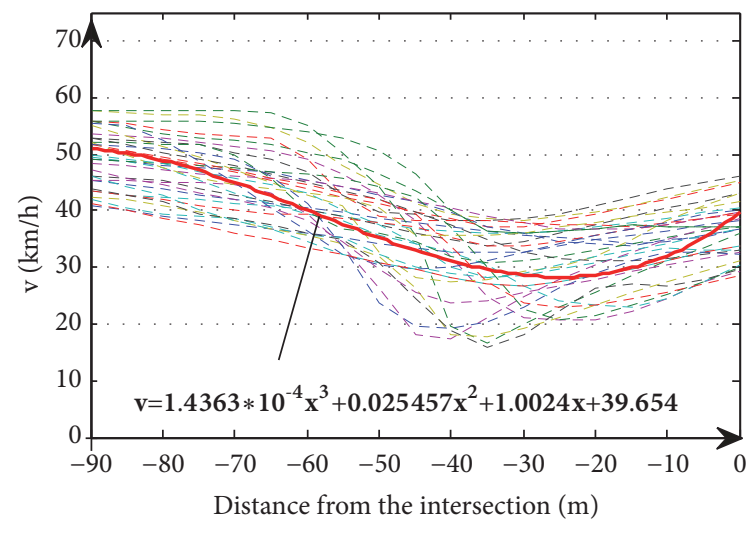

FIGURE 6: Velocity prediction curve.

deceleration phenomena. Meanwhile, when the warning system is implemented, subjects are able to perceive the danger earlier, take deceleration measures more calmly, and effectively avoid emergency braking behavior.

3.1.4. Speed Prediction Model. In order to determine the accuracy of the conflict recognition algorithm, the speed variation characteristics after installing the warning system need to be quantified. Therefore, a speed prediction model is proposed in this section.

Analysis of the change in speed implemented by drivers when the warning lights were flashing indicates that, in most cases, speed began to decline at a distance of $90 \mathrm{~m}$ from the intersection. Therefore, the fitting range of the velocity variation curve was $(-90,0)$. The fitting degree index, $R$, is a new statistical parameter used to determine the degree of fit of the nonlinear regression equation.

$$
R_{\text {new }}=1-\sqrt{\frac{Q}{\sum y^{2}}}
$$

where $\mathrm{Q}$ is residual sum of squares; $\mathrm{Q}=\sum(\mathrm{y}-\mathrm{y} *)^{2}$; $\mathrm{y}$ is actual measured value; $\mathrm{y} *$ is predictive value.

Through the analysis of the various curves, a cubic polynomial was found to have a high fitting precision $\left(\mathrm{R}_{\text {new }}\right.$ $=0.8312)$ and the regression equation was relatively simple. Therefore, a cubic polynomial regression equation was used to fit the velocity curve. The fitting results are shown in Figure 6(a).

The speed prediction model is shown in

$$
V=0.00014363 x^{3}+0.025457 x^{2}+1.0024 x+39.654,
$$

wherex is position of the vehicle from the intersection; the range of $x$ is $(-90,0)$.

3.2. Algorithm Verification. To validate the accuracy and reliability of the proposed warning algorithm, numerical simulations were carried out based on cosimulation in CarSim and Simulink.

\subsubsection{CarSim/Simulink Cosimulation}

(i) Principle. An emergency collision avoidance situation was designed in CarSim; the target vehicle was controlled by Simulink based on actual conditions. The Simulink input was the vehicle position and velocity, and the goal was to generate an output dictating when and how strongly to brake. The brake strength was determined by the velocity prediction model to ensure that the simulation was consistent with the actual situation. The warning logic of the warning system was converted into a Simulink logic diagram, as shown in Figure 7.

(ii) Scenario. In these simulations, two different scenarios were considered:

(1) No warning system: the two cars start at specified locations with a constant velocity.

(2) Set warning system: the major road vehicle decides whether or not to take deceleration measures based on the risk assessment results, and the throttle force is controlled through the speed prediction model. The minor road vehicle has no speed intervention.

3.2.2. Simulation Results Analysis. To verify the reliability of the proposed warning logic and algorithm, numerical simulations were carried out via Matlab/Simulink cosimulation. To ensure the reliability of the algorithm at different speeds and relative position conditions, different situations (Table 6) were tested separately through CarSim/Simulink cosimulations. The experiments assume that the main road vehicle starts at $150 \mathrm{~m}$ away from the intersection ( $\mathrm{X}=$ 150). According to the conflict warning threshold (see (7)), the starting point range of the minor road vehicle can be obtained. Table 6 shows the starting position of the minor road vehicle in the boundary situation $\left(\mathrm{Y}_{\min }, \mathrm{Y}_{\max }\right)$ and the most dangerous situation $\left(\mathrm{Y}_{\text {danger }}\right)$. It was verified that, within the conflict threshold, the major road driver can receive the warning signal and successfully avoid the conflict.

The following is an example $\left(V_{\mathrm{x}}=60 \mathrm{~km} / \mathrm{h}, \mathrm{V}_{\mathrm{y}}=30 \mathrm{~km} / \mathrm{h}\right)$ to describe the conflict avoidance process of the three levels of urgency $\left(\mathrm{Y}_{\text {danger }}, \mathrm{Y}_{\min }, \mathrm{Y}_{\max }\right)$.

(1) $\left(Y_{\text {danger }}\right)$ For Scenario 1, the major road vehicle maintains its initial driving velocity and collides with the minor road vehicle (Figure 8(a1)). In Scenario 2, the major road driver adjusts their brake strength according to the speed prediction model (Figure 8(a2)). After taking the collision avoidance measures, the minimum distance between the two cars increased to $9.5 \mathrm{~m}$, and the collision was effectively avoided.

(2) $\left(Y_{\min }\right)$ In Scenario 1, the minor road vehicle first arrived at the conflict point. As shown in Figure 8(b1), the minimum distance between the two vehicles was $45.4 \mathrm{~m}$; the two vehicles have no collision risk. In Scenario 2, when the major road vehicle did not significantly slow down, the minor road vehicle reached the intersection and the warning signal was lifted. Therefore, the relative distance of the two cars and the speed of the major road vehicle did not change significantly (Figure 8(b2)). 
TABLE 6: Starting position of minor road vehicle (Y).

\begin{tabular}{|c|c|c|c|c|c|c|c|c|c|c|c|c|}
\hline \multirow{2}{*}{$X=150 m$} & \multicolumn{3}{|c|}{$\mathrm{Vx}=30 \mathrm{Km} / \mathrm{h}$} & \multicolumn{3}{|c|}{$\mathrm{Vx}=40 \mathrm{Km} / \mathrm{h}$} & \multicolumn{3}{|c|}{$\mathrm{Vx}=50 \mathrm{Km} / \mathrm{h}$} & \multicolumn{3}{|c|}{$\mathrm{Vx}=60 \mathrm{Km} / \mathrm{h}$} \\
\hline & $Y_{\min }$ & $Y_{\text {danger }}$ & $Y_{\max }$ & $\mathrm{Y}_{\min }$ & $Y_{\text {danger }}$ & $Y_{\max }$ & $Y_{\min }$ & $\mathrm{Y}_{\text {danger }}$ & $\mathrm{Y}_{\max }$ & $Y_{\min }$ & $Y_{\text {danger }}$ & $\mathrm{Y}_{\max }$ \\
\hline$V_{y}=30$ & 98 & 150 & 176 & 60 & 113 & 135 & 38 & 90 & 110 & 23 & 75 & 94 \\
\hline$V_{y}=40$ & 135 & 200 & 231 & 85 & 150 & 176 & 55 & 120 & 143 & 35 & 100 & 121 \\
\hline$V_{y}=50$ & 173 & 250 & 286 & 110 & 188 & 217 & 73 & 150 & 176 & 48 & 125 & 149 \\
\hline$V_{y}=60$ & 210 & 300 & 341 & 135 & 225 & 259 & 90 & 180 & 209 & 60 & 150 & 176 \\
\hline
\end{tabular}

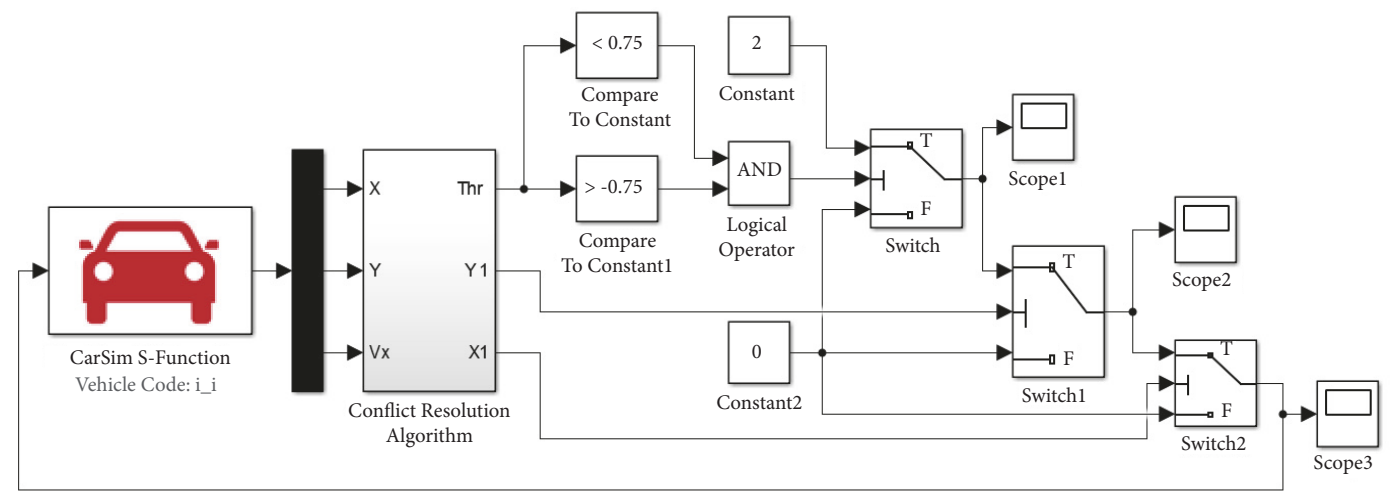

Figure 7: Simulink logic diagram.

(3) $\left(Y_{\max }\right)$ In Scenario 1, the major road vehicle first arrived at the conflict point. The minimum distance between the two vehicles was $14.3 \mathrm{~m}$; they have no collision risk (Figure 8(c1)). In Scenario 2, in order to avoid the collision with the minor road vehicle, the major road vehicle took a continuous deceleration measure, and the speed was reduced to $13.2 \mathrm{~km} / \mathrm{h}$ (Figure $8(\mathrm{c} 2)$ ). The minimum distance between the two vehicles was $10.2 \mathrm{~m}$; the major road vehicle experienced a $4 \mathrm{~s}$ intersection delay.

As can be seen from the simulation results, under the boundary conditions $\left(\mathrm{Y}_{\min }\right.$ and $\mathrm{Y}_{\max }$ ), even if the major road driver does not take deceleration measures, the two cars will not collide with each other. Therefore, the warning logic and algorithm can effectively identify almost all potential conflicts under the assumed conditions.

\section{Discussion}

In this research, the principles of the warning system are discussed in detail, including how the vehicle dynamics data are collected, and how potential collisions are identified and avoided.

This paper evaluated the effectiveness of the warning system by analyzing driving behavior, including vehicles' maneuvering performance and drivers' operating behavior under different scenarios (no warning system and presence of the warning system). Since the road environment of the two scenarios was the same, this could lead to a learning effect. In order to create an emergency collision avoidance environment and reduce the learning effect, the presence (or not) of a car driving into the intersection occurred at random. Further, the minor road adopted a "must encounter mode" with regard to passing another vehicle in the intersection; namely, no matter what speed the major road subject adopted, they always encountered a minor road car. As can be seen from Figure 5, in the no warning system scenario, the drivers showed a sudden braking phenomenon in front of the intersection, which implies that the experimental scenarios produce an emergency collision avoidance situation. Thus, a learning effect does not appear to influence the results and conclusions.

Meanwhile, the paper also validated the accuracy of the proposed conflict resolution algorithm via CarSim/Simulink cosimulation. These simulation experiments assisted with identifying timing problems and logical inconsistencies or incompleteness, without the need for expensive physical testing. As can be seen from the analysis results, the algorithm we propose can ensure safe driving on cross-intensive roads. However, the algorithm is not perfect. In order to ensure safety, the warning threshold must be larger than actually needed, which may cause a short time delay at the intersection. Therefore, choosing an appropriate conflict threshold to ensure safety, while minimizing intersection delay, is an important future research direction.

\section{Conclusions}

The warning system proposed primarily applies to crossintensive roads. In this study, the effectiveness of the system was evaluated using driving simulation experiments, and the reliability of the conflict identification algorithm was verified 


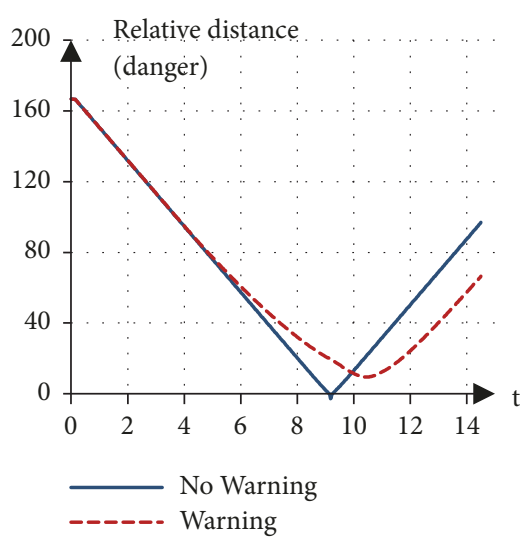

(a1)

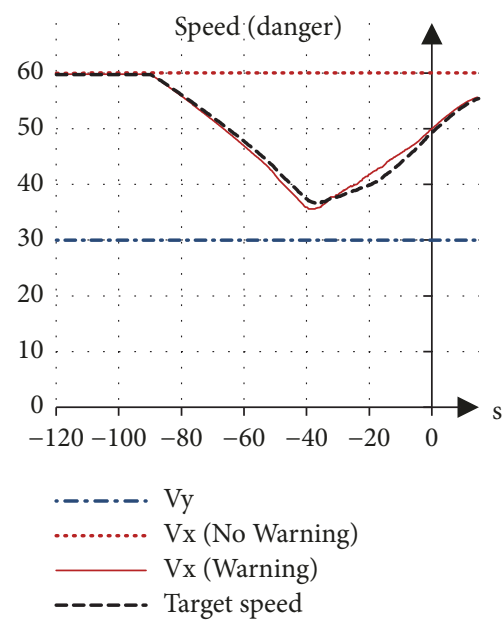

(a2)

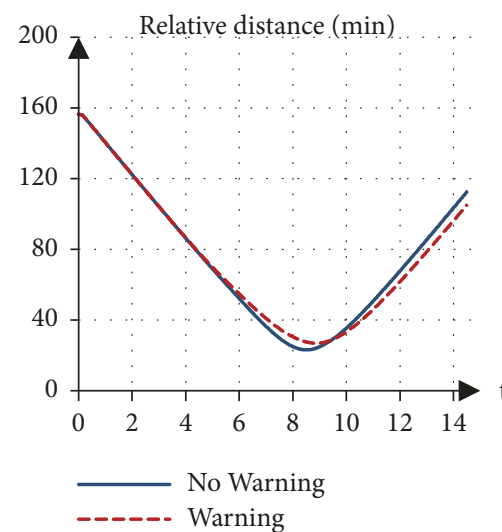

(b1)

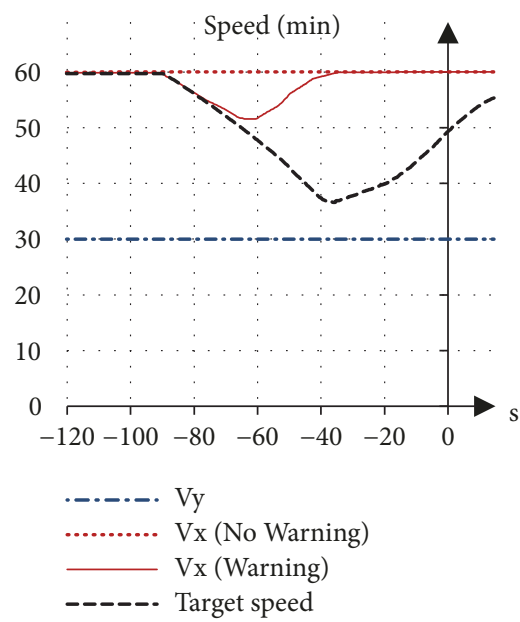

(b2)

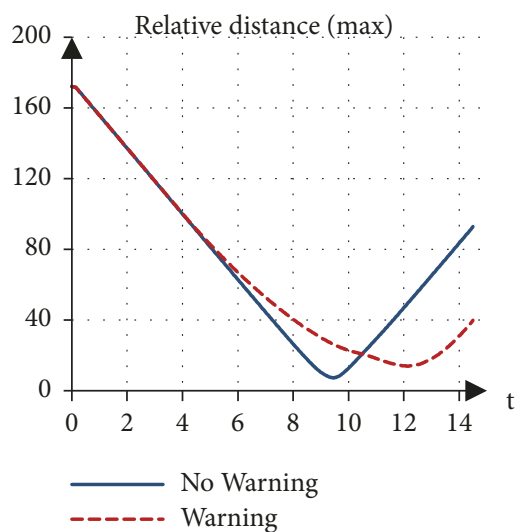

$(\mathrm{c} 1)$

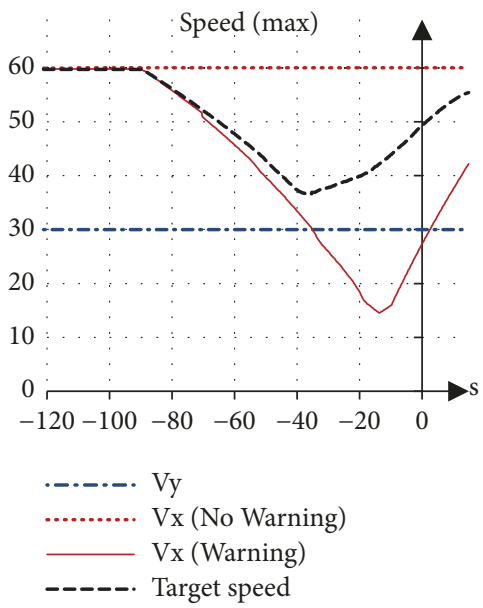

(c2)

Figure 8: Simulation results $\left(\mathrm{V}_{\mathrm{x}}=60 \mathrm{~km} / \mathrm{h}, \mathrm{Vy}=30 \mathrm{~km} / \mathrm{h}\right)$. (1) Relative distance-time curve; (2) velocity-distance curve; (a) $\mathrm{Y}_{\text {danger }}$; (b) $\mathrm{Y}_{\text {min }}$; (c) $Y_{\max } \cdot$

by numerical CarSim/Simulink cosimulations. Through experimentation, the following conclusions can be made:

(i) The proposed system can transmit the detection results in a timely manner via a roadside sensor and enables drivers to perceive conflicting vehicles in advance.

(ii) During the collision avoidance process, the warning system can cause drivers to take deceleration measures earlier, effectively avoiding emergency braking behavior and improving speed continuity and driving stability.

(iii) The speed prediction model obtained in the collision avoidance experiments had a good fit (Rnew $=$ 0.8132 ). The model can reliably quantify the driver speed change trends after installation of the warning system.

(iv) The proposed conflict recognition algorithm can effectively identify almost all potential intersection conflicts and ensures safe driving on cross-intensive roads.
In summary, the warning system proposed herein is a practical and effective system. In future research, factors such as communication delay and potential incorrect measurements by sensors should be considered. Straight-right turn conflict and multicar conflict are also scenarios that should be addressed in future research.

\section{Data Availability}

The data used to support the findings of this study are available from the corresponding author upon request.

\section{Conflicts of Interest}

The authors declare that they have no conflicts of interest.

\section{Acknowledgments}

This study was supported by a National Natural Science Foundation of China Project (51578027). 


\section{References}

[1] L. Chen and C. Englund, "Cooperative intersection management: a survey," IEEE Transactions on Intelligent Transportation Systems, vol. 17, no. 2, pp. 570-586, 2016.

[2] C. Huang, S. Lin, C. Yang, and C. Chou, "A collision prewarning algorithm based on $\mathrm{v} 2 \mathrm{v}$ communication," in Proceedings of the 4th International Conference on Ubiquitous Information, pp. 1-6, Fukuoka, Japan, December 2009.

[3] M. R. Hafner, D. Cunningham, L. Caminiti, and D. Del Vecchio, "Cooperative collision avoidance at intersections: Algorithms and experiments," IEEE Transactions on Intelligent Transportation Systems, vol. 14, no. 3, pp. 1162-1175, 2013.

[4] J. Lee and B. Park, "Development and evaluation of a cooperative vehicle intersection control algorithm under the connected vehicles environment," IEEE Transactions on Intelligent Transportation Systems, vol. 13, no. 1, pp. 81-90, 2012.

[5] I. H. Zohdy, R. K. Kamalanathsharma, and H. Rakha, "Intersection management for autonomous vehicles using iCACC," in Proceedings of the 2012 15th International IEEE Conference on Intelligent Transportation Systems, ITSC 2012, pp. 1109-1114, Anchorage, Alaska, USA, September 2012.

[6] K. C. Fuerstenberg, "A new European approach for intersection safety - The EC-project INTERSAFE," in Proceedings of the 8th International IEEE Conference on Intelligent Transportation Systems, pp. 343-347, Austria, September 2005.

[7] L. Jiang, C. Bai-Gen, W. Yun-Peng, and W. Jian, "Locationbased Cooperative Vehicle Collision Avoidance for Unsignalized Intersections: A Multi-sensor Integration Approach," in Proceedings of the 2012 International Conference on Connected Vehicles and Expo (ICCVE), pp. 246-251, Beijing, China, December 2012.

[8] C. M. Huang and S. Y. Lin, "An Advanced Vehicle Collision Warning Algorithm over the DSRC Communication Environment: An Advanced Vehicle Collision Warning Algorithm," in Proceedings of the 2013 IEEE 27th International Conference on Advanced Information Networking and Applications (AINA), pp. 696-702, Barcelona, Spain, March 2013.

[9] G. S. Aoude, V. R. Desaraju, L. H. Stephens, and J. P. How, "Driver behavior classification at intersections and validation on large naturalistic data set," IEEE Transactions on Intelligent Transportation Systems, vol. 13, no. 2, pp. 724-736, 2012.

[10] S. S. Pulugurtha, S. S. Nambisan, M. R. Dangeti, and V. Vasudevan, "In An evaluation of effectiveness of traffic signs to enhance pedestrian safety 12 ," Transportation Research Board 89th Annual Meeting, 2010.

[11] D. Sun, S. V. S. K. Ukkusuri, R. F. Benekohal, and S. T. Waller, Modeling of Motorist-Pedestrian Interaction at Uncontrolled Mid-Block Crosswalks, 2002.

[12] A. Gorjestani, A. Menon, P. M. Cheng, C. Shankwitz, and M. Donath, Determination of the alert and warning timing for the cooperative intersection collision avoidance system-stop sign assist using macroscopic and microscopic data: Cicas-ssa report \#1, University of Minnesota, Twin Cities, Minn, USA, 2010.

[13] M. Alonso, M. H. Vega, and O. Martín, "Driving simulation study for the analysis of distraction effects in longitudinal driving behaviour," Cognition, Technology \& Work, vol. 14, no. 4, pp. 283-297, 2012.

[14] A. Montella and F. Mauriello, "Drivers speed behavior at rural intersections: Simulator experiment and real world monitoring," Bulletin of the World Health Organization, vol. 83, pp. 2733, 2011.
[15] R. Van Der Horst and S. De Ridder, "Influence of roadside infrastructure on driving behavior: Driving simulator study," Transportation Research Record, no. 2018, pp. 36-44, 2007.

[16] B. Kihei, J. A. Copeland, and Y. Chang, "Doppler domain localization for collision avoidance in VANETs by using omnidirectional antennas," in Proceedings of the 3rd International Conference on Connected Vehicles and Expo, ICCVE 2014, pp. 331-337, Austria, November 2014.

[17] R. Toledo-Moreo and M. A. Zamora-Izquierdo, "Collision avoidance support in roads with lateral and longitudinal maneuver prediction by fusing GPS/IMU and digital maps," Transportation Research Part C: Emerging Technologies, vol. 18, no. 4, pp. 611-625, 2010.

[18] J. Liu, B. Cai, Y. Wang, and J. Wang, "A lane level positioningbased cooperative vehicle conflict resolution algorithm for unsignalized intersection collisions," Computers and Electrical Engineering, vol. 39, no. 5, pp. 1381-1398, 2013.

[19] A. G. Hagargund, R. Udayshankar, and N. Rashmi, "Radar based cost effective vehicle speed detection using zero cross detection," International Journal of Electrical Electronics \& Data Communication, vol. 1, 2013.

[20] J. Pan, B. Qiang, Z. Liu, Y. Hu, and S. Wang, "Fast identification and modification of angle error based on prior information of velocity-measurement radar," Lecture Notes in Electrical Engineering, vol. 187, pp. 155-163, 2013.

[21] K. B. Kahl and D. B. Fambro, "Investigation of object-related accidents affecting stopping sight distances," Transportation Research Record, no. 1500, pp. 25-30, 1995.

[22] R. Layton and K. Dixon, Stopping sight distance, 2015.

[23] T. Kanungo, D. M. Mount, N. S. Netanyahu, C. D. Piatko, R. Silverman, and A. Y. Wu, "An efficient k-means clustering algorithms: analysis and implementation," IEEE Transactions on Pattern Analysis and Machine Intelligence, vol. 24, no. 7, pp. 881$892,2002$. 


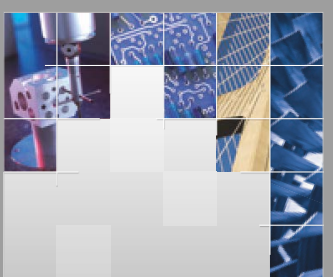

\section{Enfincering}
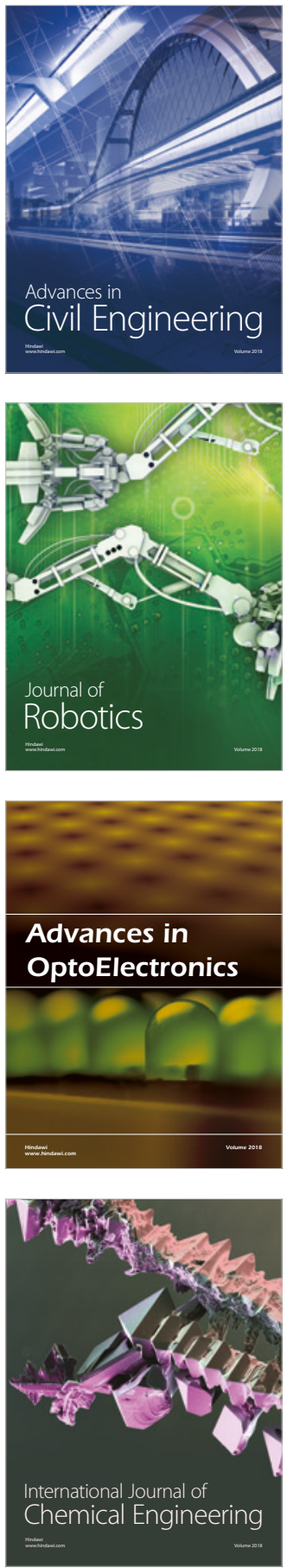

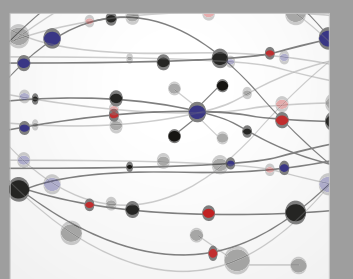

\section{Rotating \\ Machinery}

The Scientific World Journal

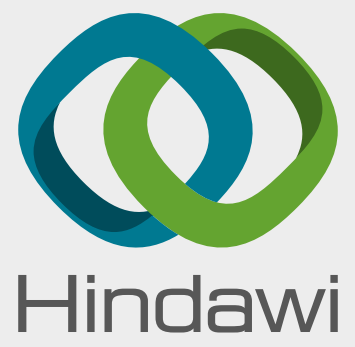

Submit your manuscripts at

www.hindawi.com
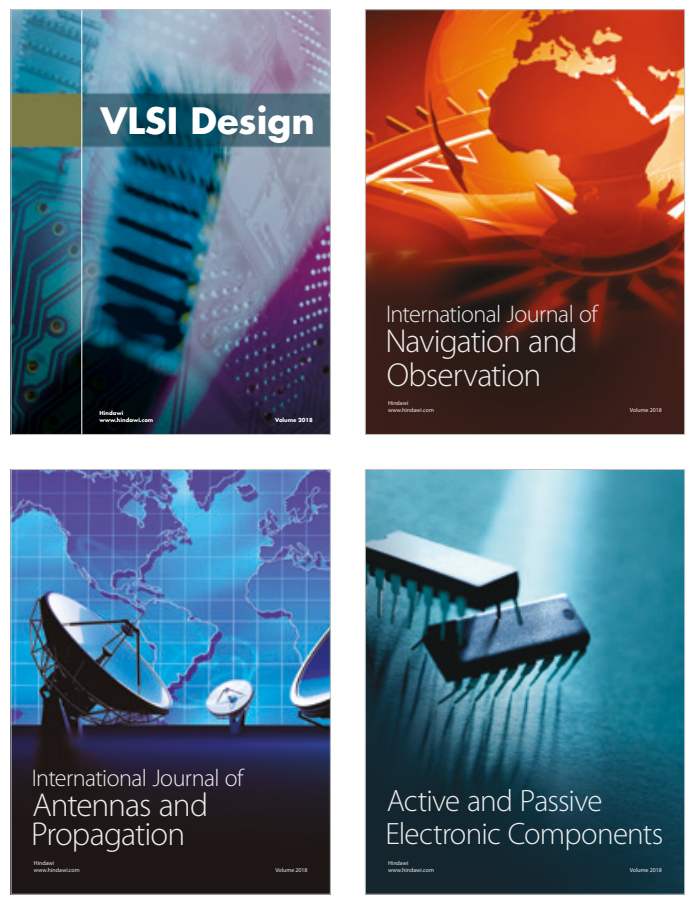
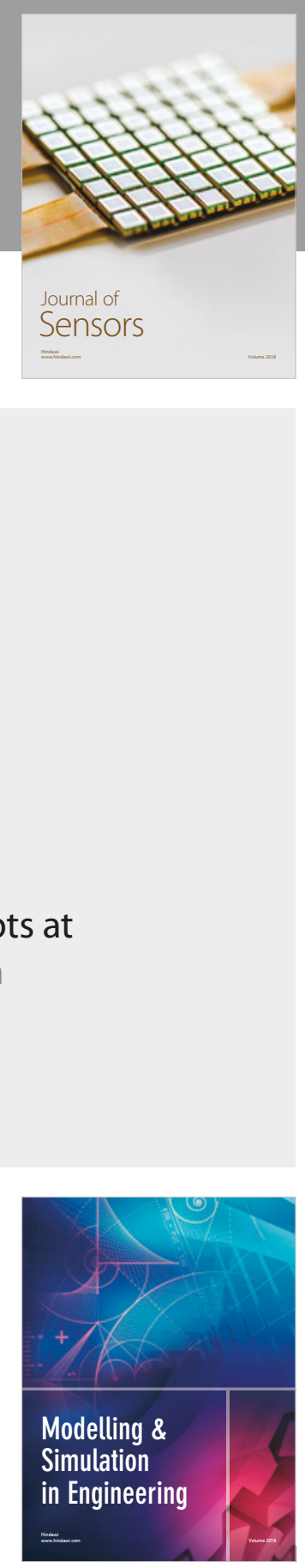

\section{Advances \\ Multimedia}
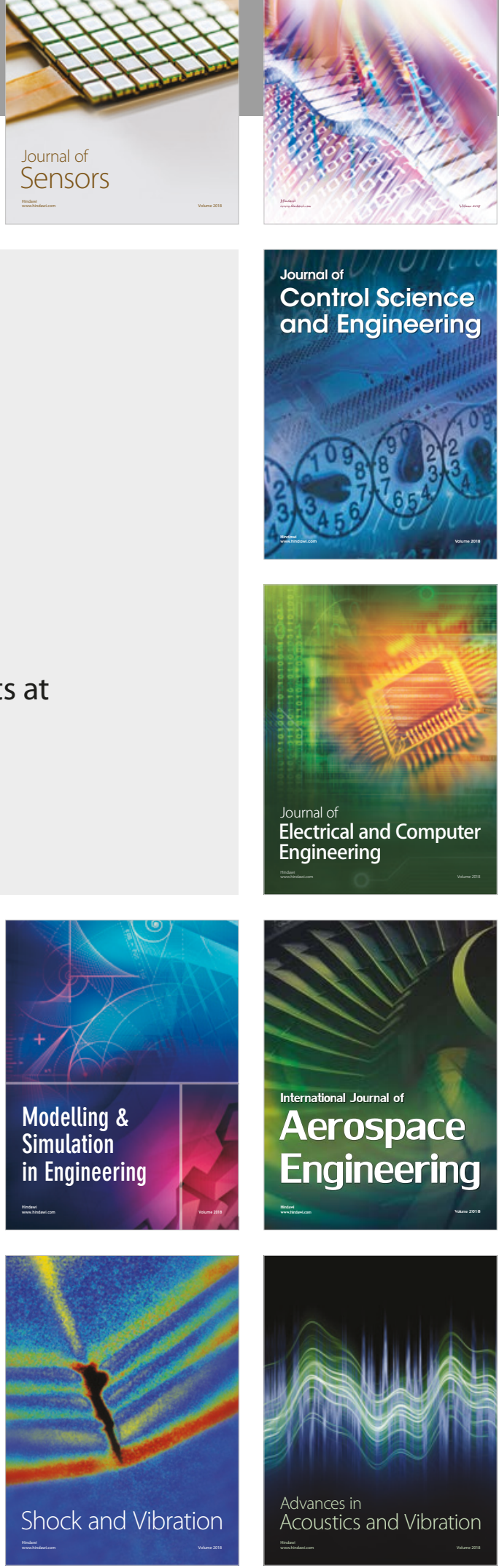